J. Clin. Chem. Clin. Biochem.

Vol. 28,1990 , pp. $527-531$

(C) 1990 Walter de Gruyter \& Co.

Berlin · New York

\title{
Nichtenzymatische Glykierung von Humanserumalbumin: Einfluß auf die Bindungskinetik an der Benzodiazepinbindungsstelle ${ }^{1}$ )
}

\author{
Von W. Wörner, S. Pfleiderer, W. Kratzer und N. Rietbrock
}

Abteilung für Klinische Pharmakologie am Klinikum der Johann Wolfgang Goethe-Universität Frankfurt am Main

(Eingegangen am 28. November 1989/27. März 1990)

Zusammenfassung: Humanserumalbumin wurde in vitro nichtenzymatisch glykiert und der Glykierungsgrad mit einem affinitätschromatographischen Verfahren bestimmt. Der Einfluß der Glykierung auf die Bindung des Modelliganden Dansylsarkosin an der Benzodiazepin-Bindungsstelle wurde mit Hilfe der Stopped-FlowMethode über die Aufzeichnung der Fluoreszenz-Zeitverläufe während des Bindungsvorganges gemessen. Die Assoziationsgeschwindigkeitskonstante $\mathrm{k}_{2}$ nahm mit steigendem Glykierungsgrad von $533,3 \mathrm{~s}^{-1}$ bei einem Anteil am Gesamtserumalbumin von 0,048 glykiertem Albumin auf $218,1 \mathrm{~s}^{-1}$ bei 0,158 glykiertem Albumin ab. Analog verhielt sich die Affinitätskonstante $\mathrm{K}_{\mathrm{A}}: 7,61 \cdot 10^{5} \mathrm{l} / \mathrm{mol}$ für Glykoalbumin $=0,048$ und 2,60 $10^{5}$ $1 / \mathrm{mol}$ für Glykoalbumin $=0,158$. Die Dissoziationsgeschwindigkeitskonstante $\mathrm{k}_{-2}$ stieg von $17,3 \mathrm{~s}^{-1}$ (glykiertes Albumin $=0,048$ ) auf $19,8 \mathrm{~s}^{-1}$ (glykiertes Albumin $=0,158$ ). Die Hemmung der Bindung erfolgt wahrscheinlich über einen allosterischen Mechanismus.

Non-enzymatic glycation of human serum albumin: Influence on the binding kinetics of the benzodiazepine binding site

Summary: Human serum albumin was non-enzymatically glycated in vitro and the glycation rate determined using an affinity chromatography method. The influence of glycation on the binding of the model ligand, dansylsarcosine, at the benzodiazepine binding site was determined with a stopped-flow method. Fluorescence time curves were recorded during the binding process. As the glycation rate increased, the association velocity constant, $\mathrm{k}_{2}$, decreased from $533.3 \mathrm{~s}^{-1}$ (glycated albumin 0.048 of total serum albumin) to $218.1 \mathrm{~s}^{-1}$ (glycated albumin 0.158 of total serum albumin). The affinity constant, $\mathrm{K}_{\mathrm{A}}$, showed a corresponding decrease from $7.61 \cdot 10^{5} \mathrm{l} / \mathrm{mol}$ (fraction of glycated albumin 0.048 ) to $2.60 \cdot 10^{5} \mathrm{l} / \mathrm{mol}$ (fraction of glycated albumin 0.158 ). The dissociation velocity constant, however, increased from $17.3 \mathrm{~s}^{-1}$ (fraction of glycated albumin 0.048 ) to $19.8 \mathrm{~s}^{-1}$ (fraction of glycated albumin 0.158 ). The inhibition of binding probably occurs via an allosteric mechanism.

\section{Einführung}

Erhöhte Konzentrationen an freier Glucose im Serum beim Diabetiker führen zu einer vermehrten Glykierung von gelösten und membranständigen Proteinen.

1) Teile der Arbeit wurden im Mai 1989 auf dem Symposium der Deutschen Gesellschaft für Pharmakologie und Toxikologie in Rauischholzhausen und im September 1989 auf der Herbsttagung der Deutschen Gesellschaft für Pharmakologie und Toxikologie in Köln vorgestellt.
Die kovalente Verknüpfung der freien Glucose mit basischen Aminosäureseitenketten erfolgt nichtenzymatisch über eine Maillard-Reaktion unter Ausbildung einer instabilen Schiff'schen Base, die sich über eine Amadori-Umlagerung in ein stabiles Fruktosamin umwandelt. Für viele dieser glykierten Proteine (Hämoglobin, Kollagen, Lipoproteine u. a. (1)) sind zum Teil erhebliche Veränderungen ihrer Eigenschaften beschrieben worden. Es erscheint daher von besonderem 
Interesse, auch beim Serumalbumin zu untersuchen, ob dieses durch Glykierungsprozesse in seiner klinisch-pharmakologisch wichtigsten Funktion - der Bindungsfähigkeit für saure und neutrale Substanzen - eingeschränkt ist. Leider existieren hierzu bisher nur einige vereinzelte, zum Teil widersprüchliche Untersuchungen.

Primärer Angriffspunkt der nichtenzymatischen Glykierung am Humanserumalbumin ist Lysin 525, doch wurden auch andere Bindungsstellen nachgewiesen (2). Wie durch Fluoreszenzmessungen gezeigt werden konnte, kommt es hierbei zu einer Konformationsänderung des Albumins, was wiederum zu einem veränderten Bindungsverhalten gegenüber bestimmten endogenen und exogenen Liganden führt (3). Von Ruiz-Cabello (4) wurde ein Einfluß der Glykierung auf die Bindung von Sulfisoxazol (Bindungsstelle I), nicht jedoch von Diazepam (Bindungsstelle II) nachgewiesen. Mit in vitro glykiertem Albumin konnte jedoch eine verminderte Bindung von Dansylsarkosin an der Bindungsstelle II festgestellt werden $(5,6)$. Es ist daher angebracht, den Bindungsverlauf eines $\mathrm{Li}$ ganden an der Bindungsstelle II bei unterschiedlich stark glykiertem Albumin mit einer geeigneten Methode $\mathrm{zu}$ verfolgen, um gesicherte Aussagen über die Konsequenzen dieser kovalenten Modifizierung treffen zu können.

\section{Material und Methoden}

In vitro-Synthese von glykiertem Serumalbumin

Gereinigtes, fettsäurefreies Humanserumalbumin (Sigma, Deisenhofen) wurde in $0,1 \mathrm{~mol} / 1$ Sörensen-Phosphatpuffer $\mathrm{pH}=7,4$ gelöst $(40 \mathrm{~g} / \mathrm{l})$, mit $D$-Glucose in Konzentrationen von $8,3 \mathrm{mmol} / 1(150 \mathrm{mg} / \mathrm{dl}), 16,7 \mathrm{mmol} / \mathrm{l}(300 \mathrm{mg} / \mathrm{dl})$ und 27,8 $\mathrm{mmol} / 1(500 \mathrm{mg} / \mathrm{dl})$ versetzt und anschließend bei $37^{\circ} \mathrm{C} 48 \mathrm{~h}$ inkubiert. Nach 6, 10, 24 und $48 \mathrm{~h}$ wurden jeweils Proben entnommen und dialysiert. Diese Proben sowie eine Albuminlösung ohne Glucosezusatz wurden nach Bestimmung des glykierten Serumalbumins zur Durchführung der Bindungsexperimente verwendet.

\section{Bestimmung des glykierten Albumins}

Die Bestimmung der glykierten Serumproteinfraktion erfolgte durch Affinitätschromatographie mit $m$-Aminophenylboratagarose (Sigma, Deisenhofen) als stationärer Phase. Zur Äquilibrierung der Säule und zur Elution der nichtglykierten Proteine diente $280 \mathrm{mmol} / \mathrm{l}$ Acetatpuffer $\mathrm{pH}=8,5$ (Waschpuffer). Die glykierten Proteine des Serums wurden mit $18 \mathrm{mmol} / \mathrm{l}$ Citratpuffer $\mathrm{pH}=4,6$ eluiert. Die Eluate wurden anschließend eingeengt und bis zur Messung bei $-20^{\circ} \mathrm{C}$ eingefroren.

Anschließend wurde die Konzentration des glykierten Humanserumalbumins photometrisch bei $620 \mathrm{~nm}$ mit Bromkresolgrün bestimmt. Der Zusammenhang zwischen Extinktionsdifferenz und Konzentration verläuft unterhalb einer Konzentration von $10 \mathrm{~g} / \mathrm{l}$ Glykoalbumin nichtlinear, so daß zuvor mit einer Verdünnungsreihe eine Kalibrierfunktion erstellt wurde.
Die Präzision in Serie für Isolierung und Bestimmung von glykiertem und nichtglykiertem Albumin ist durch einen Variationskoeffizienten von $6,70 \%(n=12)$ für die nichtglykierte und von $8,33 \%(\mathrm{n}=12)$ für die glykierte Fraktion charakterisiert. Die Wiederfindung betrug $97,6 \%$.

\section{Durchführung der kinetischen Messungen}

Die kinetischen Experimente wurden mit einer Durrum-Gibson Stopped Flow-Apparatur bei einer konstanten Temperatur von $25^{\circ} \mathrm{C}$ durchgeführt $(7,8)$. Als fluoreszierender Marker diente Dansylsarkosin $(2,5 \mu \mathrm{mol} / \mathrm{l})$, das spezifisch an die Benzodiazepin-Bindungsstelle zu binden vermag (Albuminkonzentration: 3 bis $109 \mu \mathrm{mol} / 1$ ). Die Intensitätsänderung der Fluoreszenz (Relaxation) wurde während der Bildung des Albumin-Dansylsarkosin-Komplexes aufgezeichnet. Die Exzitation erfolgte bei einer Wellenlänge von $351 \mathrm{~nm}$, die Emission wurde bei 475 nm gemessen.

Bei jedem Bindungsexperiment wurden vier reproduzierbare Fluoreszenzzeitverläufe aufgezeichnet und zu zwei Paaren summiert. Die Signale wurden auf einen Personalcomputer (Wang Instr.) übertragen und mit Hilfe der Rotating Iterative Procedure (9) an vorgegebene Exponentialtherme angepaßt. Nach Bernasconi (10) besteht zwischen der beobachteten Relaxationskonstanten und den kinetischen Konstanten folgender linearer Zusammenhang:

$$
\mathrm{k}_{\mathrm{rel}}=\mathrm{k}_{2} \cdot \frac{\mathrm{K}_{\mathrm{A}^{\prime}} \cdot\left(\mathrm{c}_{\mathrm{DS}}+\mathrm{c}_{\mathrm{HSA}}\right)}{1+\mathrm{K}_{\mathrm{A}^{\prime}} \cdot\left(\mathrm{c}_{\mathrm{DS}}+\mathrm{c}_{\mathrm{HSA}}\right)}+\mathrm{k}_{-2}
$$

$\mathrm{k}_{2}$ : $\quad$ Assoziationsgeschwindigkeitskonstante

$\mathrm{k}_{-2}$ : Dissoziationsgeschwindigkeitskonstante

$\mathrm{K}_{\mathrm{A}^{\prime}}$ : $\quad$ Affinitätskonstante der unspezifischen Bindung

$\mathrm{k}_{\mathrm{rel}}$ : $\quad$ beobachtete Relaxationskonstante

$\mathrm{c}_{\mathrm{DS}}, \mathrm{c}_{\mathrm{HSA}}$ : Konzentration von Dansylsarkosin und Humanserumalbumin im Bindungsgleichgewicht

Unter Verwendung der Konzentrationsverhältnisse vor der Reaktion und der Relaxationskonstanten wurden anschließend die kinetischen Konstanten mit Hilfe der Methode der kleinsten Fehlerquadratsummen angepaßt.

$$
\mathrm{K}_{\mathrm{A}}=\mathrm{K}_{\mathrm{A}^{\prime}} \cdot \frac{\mathrm{k}_{2}}{\mathrm{k}_{-2}}
$$

\section{$\mathrm{K}_{\mathrm{A}}$ : Affinitätskonstante}

Außerdem wurden Dissoziationsexperimente mit $500 \mu \mathrm{mol} / 1$ Ibuprofen, welches Dansylsarkosin unter Fluoreszenzminderung aus seiner Bindung zu verdrängen vermag, durchgeführt.

\section{Ergebnisse}

Der Grad der Glykierung des Albumins korreliert sowohl mit der eingesetzten Glucosekonzentration als auch mit der Inkubationszeit $(\mathrm{r}=0,897 / \mathrm{p}<0,01)$ (Tab. 1). In allen drei Versuchsansätzen steigt der Gehalt an glykiertem Albumin mit zunehmender Inkubationsdauer um das Zwei- bis Dreifache an; es werden pro Zeitintervall um so höhere Konzentrationen erreicht, je mehr Glucose eingesetzt worden ist.

Die Assoziationsgeschwindigkeitskonstante $\mathrm{k}_{2}$ (Abb. 1,2) für die Bindung von Dansylsarkosin an Albumin nimmt mit steigendem Glykierungsgrad um $60 \%$ von 
Tab. 1. Anteil von glykiertem Serumalbumin am Gesamtalbumin während 48stündiger Inkubation mit unterschiedlichen Glucosekonzentrationen

\begin{tabular}{llllll}
\hline $\begin{array}{l}\text { Glucose } \\
(\mathrm{mmol} / \mathrm{l})\end{array}$ & \multicolumn{2}{l}{ Zeit $(\mathrm{h})$} & & & \\
\cline { 2 - 6 } & 0 & 6 & 10 & 24 & 48 \\
\hline 8,3 & 0,048 & 0,063 & 0,070 & 0,108 & 0,117 \\
16,7 & 0,048 & 0,057 & 0,080 & 0,121 & 0,152 \\
27,8 & 0,048 & 0,069 & 0,098 & 0,147 & 0,158 \\
\hline
\end{tabular}

$533,3 \mathrm{~s}^{-1}$ (Anteil an glykiertem Albumin $=0,048$ ) auf $218,2 \mathrm{~s}^{-1}$ (glykiertes Albumin $\left.=0,158\right)$ ab $(\mathrm{r}=0,925$ / $\mathrm{p}<0,001)$.

Die Dissoziationsgeschwindigkeitskonstante $\mathrm{k}_{-2}$ steigt dagegen langsam, aber signifikant $(r=0,700 /$ $\mathrm{p}<0,05)$ mit zunehmender Glykierung von $17,3 \mathrm{~s}^{-1}$ (glykiertes Albumin $=0,048$ ) auf $19,8 \mathrm{~s}^{-1}$ (glykiertes Albumin $=0,158$ ).

Die Affinitätskonstante $\mathrm{K}_{\mathrm{A}^{\prime}}$ für die unspezifische Bindung von Dansylsarkosin ist mit einem Mittelwert von $2,48 \cdot 10^{4} \pm 0,42 \cdot 10^{4} 1 / \mathrm{mol}$ unabhängig vom Ausma $ß$ der Glykierung.

Die Gesamtaffinitätskonstante $\mathrm{K}_{\mathrm{A}}$ (Abb. 3, 4) hingegen nimmt analog zur Assoziationsgeschwindigkeit

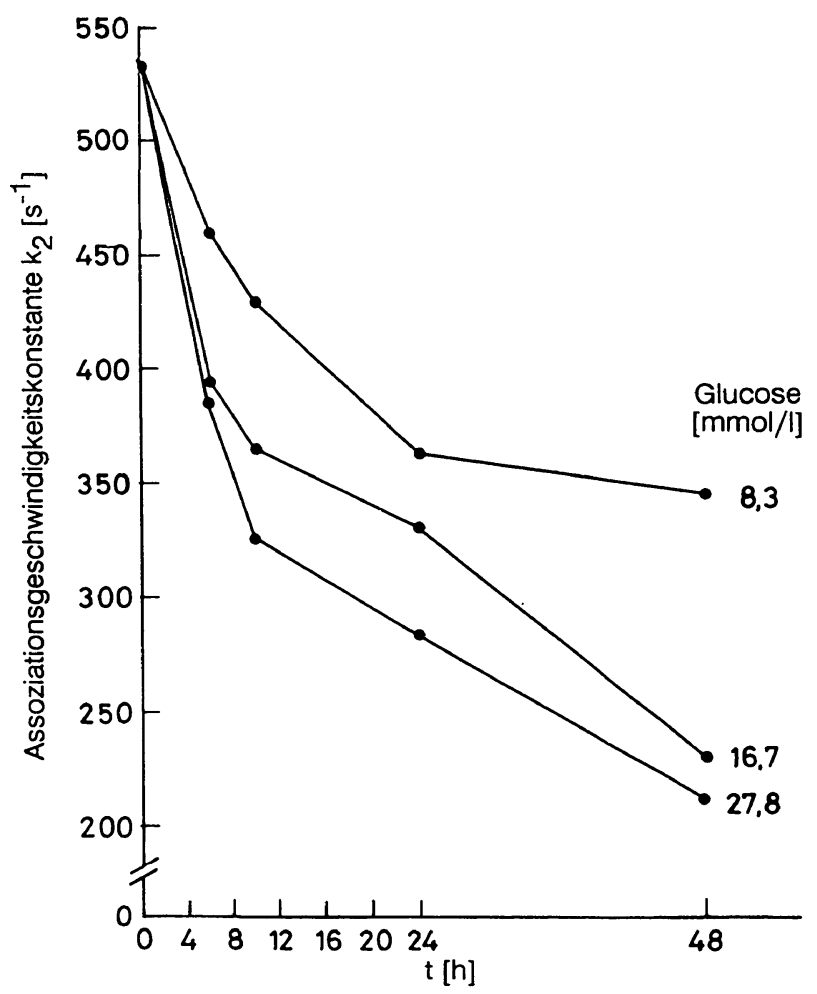

Abb. 1. Assoziationsgeschwindigkeitskonstante $k_{2}$ von Dansylsarkosin während einer 48stündigen Inkubation mit verschiedenen Glucosekonzentrationen signifikant $(r=0,780 / p<0,01)$ mit steigendem Glykierungsgrad um zwei Drittel von $7,61 \cdot 10^{5} 1 / \mathrm{mol}$ (glykiertes Albumin = 0,048) auf 2,60 $10^{5} 1 / \mathrm{mol}$ (glykiertes Albumin $=0,158) \mathrm{ab}$.

Es konnte gezeigt werden, daß hinsichtlich der Komplexierung mit Bromkresolgrün und des Absorptionsverhaltens bei $280 \mathrm{~nm}$ kein Unterschied zwischen glykiertem und nichtglykiertem Albumin besteht.

\section{Diskussion}

Mit den beschriebenen Untersuchungen konnte eindeutig gezeigt werden, daß die in der Folge einer erhöhten Blutzuckerkonzentration gesteigerte nichtenzymatische Glykierung zu einer Hemmung der Bindung an der Bindungsstelle II von Humanserumalbumin führt. Die von uns in vitro gefundenen synthetisierten Glykoalbumin-Konzentrationen repräsentieren Werte, wie sie auch bei diabetischen Patienten auftreten (11).

Die Bildung des glykierten Albumins läßt sich nicht mit der von Baynes (12) gefundenen Kinetik in Übereinstimmung bringen. Die Glykierungsrate beträgt unter den in dieser Arbeit gewählten Bedingungen 0,06 Anteile vom Albumin für die ersten $24 \mathrm{~h}$ Inkubation mit $8,8 \mathrm{mmol} /$ Glucose, d. h. $0,0072 / \mathrm{Tag} \cdot \mathrm{mmol}$

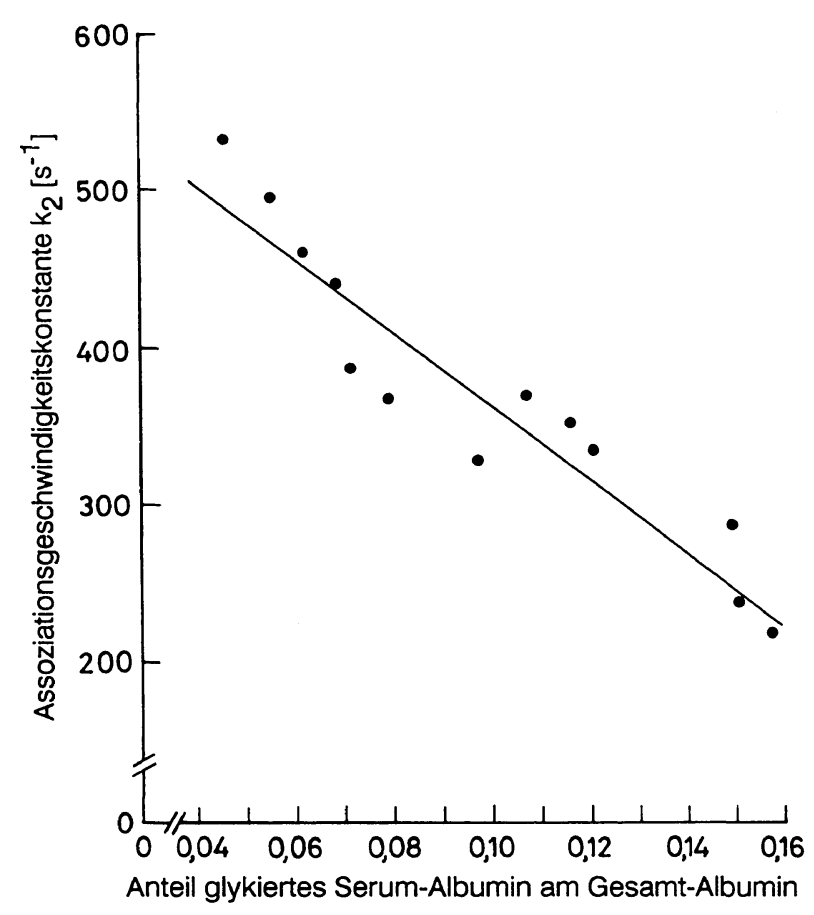

Abb. 2. Assoziationsgeschwindigkeitskonstante $\mathrm{k}_{2}$ von Dansylsarkosin in Abhängigkeit vom Anteil an glykiertem Albumin am Gesamt-Albumin $(y=-23,6 \cdot x+602,5$ $/ \mathrm{r}=0,925 / \mathrm{p}<0,001)$ 


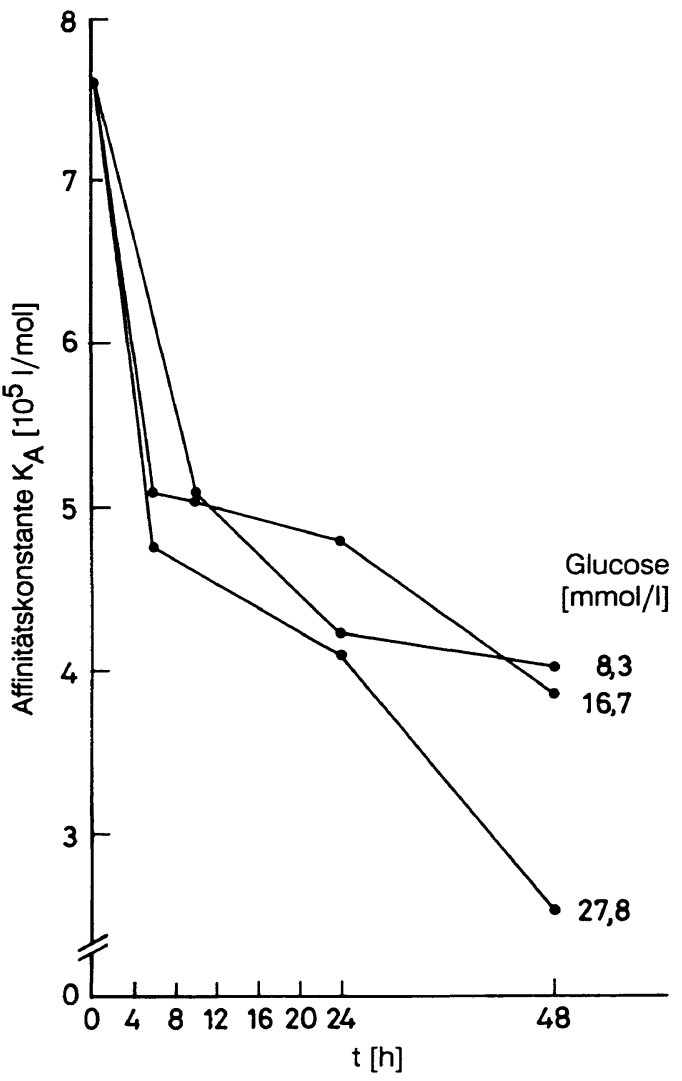

Abb. 3. Affinitätskonstante $\mathrm{K}_{\mathrm{A}}$ von Dansylsarkosin während einer 48stündigen Inkubation mit verschiedenen Glucosekonzentrationen

Glucose. Baynes fand hingegen eine Glykierungsrate von 0,013 des Albumins bei $24 \mathrm{~h}$ Inkubation mit 5,5 mmol Glucose, was einer Geschwindigkeit von $0,0025 / \mathrm{Tag} \cdot \mathrm{mmol}$ Glucose entspricht. Diese zunächst überraschende Tatsache findet ihre Erklärung darin, daß Baynes die Gesamtmenge der pro Zeiteinheit inkorporierten Glucose bestimmt hat, während in der vorliegenden Arbeit die Konzentration des glykierten Albumins - unabhängig von der Anzahl der gebundenen Glucosemoleküle - gemessen wurde. Außerdem sind Geschwindigkeit und Ausmaß der Glykierung abhängig von der Albuminkonzentration und der Anwesenheit freier Fettsäuren.

Die Verwendung von gereinigtem, fettsäurefreien Albumin war erforderlich, um eine Überlagerung der durch nichtenzymatische Glykierung induzierten Effekte durch Interaktionen der bei Diabetikern oftmals erhöhten freien Fettsäuren zu vermeiden (13). Zum Studium der Benzodiazepinbindung wurde mit dem Liganden Dansylsarkosin gearbeitet, da Benzodiazepine selbst wegen ihrer fehlenden Eigenfluoreszenz für die beschriebenen Experimente nicht geeignet sind. Die verwendete Modellsubstanz bindet jedoch ausschließlich an der Benzodiazepin-Bindungsstelle und wurde vielfach zur Charakterisierung dieser Region eingesetzt $(13,14)$.

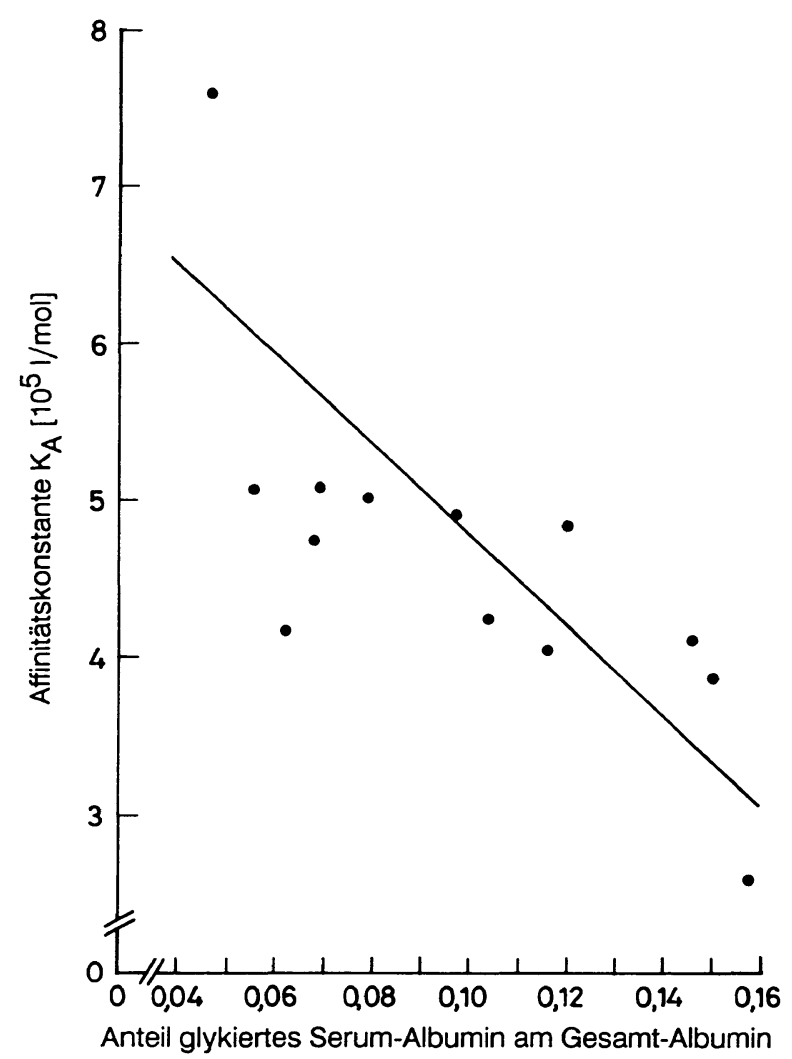

Abb. 4. Affinitätskonstante $\mathrm{K}_{\mathrm{A}}$ von Dansylsarkosin in Abhängigkeit vom Anteil an glykiertem Albumin am GesamtAlbumin $(y=-0,29 \cdot x+7,73 / r=0,780 /$ $\mathrm{p}<0,001)$

Die Bindungshemmung beruht vor allem auf einer Verminderung der Assoziationsgeschwindigkeit und nur in geringem Maße auf einer Erhöhung der Dissoziationsgeschwindigkeit. Die Konstanz der Affinitätskonstante für die unspezifische Bindung zeigt, daß analog zur allosterischen Hemmung der Dansylsarkosin-Bindung durch freie Fettsäuren die unspezifische Anlagerung des Liganden an die Oberfläche des Albuminmoleküls nicht behindert ist. Erst die mit einer konformativen Änderung verbundene Bildung des stabilen Albumin-Liganden-Komplexes erfolgt stark verlangsamt.

Der Mechanismus der hemmenden Wirkung von Glykierungen auf die Albuminbindung von Pharmaka beruht wahrscheinlich zum Großteil auf einer Änderung der Tertiärstruktur des Albumins. Eine Konformationsänderung infolge einer Glykierung am Lysin 525 wurde durch Messungen der Fluoreszenzemission des einzelnen Tryptophanrestes 214 nachgewiesen, welche eine $30 \%$ ige Intensitätsminderung sowie eine Verschiebung zu kürzeren Wellenlängen, verglichen mit nativem Albumin, aufweist (3). Der Beweis, daß sich diese konformative Alteration auch auf die Bindungsstelle II erstreckt, wird durch die Beobachtung einer mit steigendem Glykierungsgrad zunehmenden Dissoziationsgeschwindigkeitskonstanten erbracht. 
Eine kompetitive Hemmung sollte sich nämlich in einer vom Ausmaß der Glykierung unabhängigen, unveränderlichen Konstanten zeigen. Allerdings ist bei sehr hohen Glucosekonzentrationen auch eine direkte Modifizierung der Bindungsstelle denkbar, da zwei potentielle Angriffspunkte für Glykierungen (Lys 199, Lys 281) (2) Bestandteile dieser Bindungsregion sind.

Aus den hier in vitro gefundenen Ergebnissen läßt sich jedoch nicht zwangsläufig eine in vivo Erhöhung des Anteils freier Benzodiazepine ableiten. Eine vermehrte Freisetzung von Benzodiazepinen aus der Eiweißbindung wird im allgemeinen durch eine entsprechende Steigerung der hepatischen Clearance ausge- glichen (15). Auswirkungen auf den therapeutischen Einsatz dieser Tranquillantien sind daher nur bei gleichzeitig eingeschränkter Leberfunktion zu erwarten. Da zudem oftmals ein reduziertes Verteilungsvolumen und eine verminderte Albuminkonzentration im Plasma vorliegen, kann eine Veränderung aus der Eiweißbindung, die in anderen Fällen leicht kompensiert werden würde, hier gravierende Folgen nach sich ziehen. Eine Dosis-Reduktion wäre somit zur Vermeidung von Überdosierungen und Kumulation des Arzneistoffes angezeigt. Allerdings scheint Albumin in vivo teilweise an anderen Stellen glykiert zu werden als in vitro (1). Zur abschließenden Klärung dieses Sachverhaltes werden daher Folgeuntersuchungen mit Seren diabetischer Patienten angestrebt.

\section{Literatur}

1. Cohen, M. P. (1986) Diabetes and Protein Glycosylation, Springer-Verlag, New York.

2. Iberg, N. \& Flückiger, N. (1986) Nonenzymatic Glycosylation of Albumin in Vivo: Identification of Multiple Glycosylated Sites. J. Biol. Chem. 261, 13542-13545.

3. Shaklai, N., Garlick, R. L. \& Bunn, H. F. (1984) Nonenzymatic Glycosylation of Human Serum Albumin Alters Its Conformation and Function. J. Biol. Chem. 259, 38123817.

4. Ruiz-Cabello, F. \& Erill, S. (1984) Abnormal serum protein binding of acidic drugs in diabetes mellitus. Clin. Pharmacol. Ther. 36, 691-695.

5. Wörner, W. (1989) Hemmung der Pharmakon-Eiweißbindung an der Benzodiazepin-Bindungsstelle von Humanserumalbumin durch nicht-kovalent und kovalent gebundene endogene Liganden, Dissertation, Frankfurt am Main.

6. Pfleiderer, S., Wörner, W. \& Rietbrock, N. (1989) Does the non-enzymatic serum albumin glycosylation inhibit benzodiazepine binding? Evidence from fast reaction binding studies in vitro. Europ. J. Clin. Pharmacol. 36, Suppl.

7. Rietbrock, N. \& Laßmann, A. (1980) Stopped-flow studies on drug protein binding. Naunyn-Schmiedeberg's Arch Pharmacol. 313, 269-274.

8. Laßmann, A. \& Rietbrock, N. (1982) Stopped-Flow Studies on Drug Protein Binding. Naunyn-Schmiedeberg's Arch. Pharmacol. 320, 185-195.

9. Von Hattingberg, H. M., Brockmeier, D. \& Kreuter, G. (1977) A rotating iterative procedure (RIP) for estimating hybrid constants in multi-compartment analysis on deskcomputers. Europ. J. Clin. Pharmacol. 11, 381-388.

10. Bernasconi, C. F. (1976) Relaxation Kinetics, Academic Press, New York.

11. Wörner, W., Pfleiderer, S., Kratzer, W., Oremek, G. \& Rietbrock, N. (1989) Selective Determination of Glycosylated Human Serum Albumin as a Parameter of MediumTerm Diabetic Control. Europ. J. Clin. Pharmacol. 36 Suppl.

12. Baynes, J. W., Thorpe, S. R., Murtiashaw, M. H. (1984) Nonenzymatic Glucosylation of Lysine Residues in Albumin. Meth. Enzymol. 106, 88-98.

13. Menke, G., Wörner, W., Kratzer, W. \& Rietbrock, N. (1989) Kinetics of drug binding to human serum albumin: allosteric and competitive inhibition at the benzodiazepine binding site by free fatty acids of various chain lengths. NaunynSchmiedeberg's Arch. Pharmacol. 339, 42-47.

14. Menke, G., Pfister, P., Sauerwein, S., Rietbrock, I., Woodcock, B. G. \& Rietbrock, N. (1987) Age-dependence and free fatty acid modulation of binding kinetics at the benzodiazepine binding site of serum albumin in neonates and adults determined using fast reaction methods. Br. J. Clin. Pharmacol. 23, 439-445.

15. Rowland, M. (1984) Protein binding and drug clearance. Clin. Pharmacokin. 9, 10-17.

Prof. Dr. N. Rietbrock

Abteilung für Klinische Pharmakologie

Universitätsklinikum Frankfurt

Theodor-Stern-Kai 7

D-6000 Frankfurt am Main 70 
